\title{
Preliminary results of EMG-based hand gestures for long term use
}

\author{
Peter Boyd, Yinfeng Fang, and Honghai Liu \\ School of Computing, University of Portsmouth, Portsmouth, United Kingdom \\ \{peter.boyd, yinfeng.fang, honghai.liu\}@port.ac.uk
}

\begin{abstract}
The application of pattern recognition techniques to Electromyography (EMG) signals has shown great potential for robust, natural, prostheses control. Despite promising development in EMG pattern recognition techniques, the non-stationary properties of these signals may render these techniques ineffective after a period of time, subsequently demanding frequent recalibration during long term use. Potentially one method to reduce the impact of non-stationary traits of EMG signals is through attempting to construct a training dataset that represents this gradual change in the signal. In this paper, we investigate the potential impact of data selection schemes for inter-day motion recognition, across a period of five days of high density data recording with an LDA classifier, and present our preliminary findings. This paper proves that training a classifier with data from several spaced points of a single day can improve its inter-day performance which subsequently supports the long term use of prosthesis. Therefore the work presented here may aid in furthering our understanding of the physiological changes in EMG signals and how they may be exploited to further improve the robustness of pattern recognition methods for long term use.
\end{abstract}

Keywords: Surface Electromyography (sEMG), Hand Gesture Recognition, Dataset Optimisation, Prosthesis, Robustness, Pattern recognition

\section{Introduction}

Across a given period of time, classification error can be expected to increase. Unfortunately, the exact reason for the increase in classification error is not properly understood, pushing researchers to attempt to quantify the shift in EMG data and classification error across time [1]. Kaufmann [11] compared how classification accuracy degraded when training a classifier with early data sets, recent datasets, and gradually updated datasets. It was suggested that there exist some variable components within EMG signals, based on the degrading accuracy over time. If it can be assumed that there exists variable components of the EMG signal, there exists potential to construct a dataset that can reasonably predict this change, whilst remaining computationally efficient.

All of these traits are natural during daily use of a prosthesis, and therefore likely to cause classification failures during long periods of usage. Through 
recalibration, the effective usage of a prosthesis can be extended. There main methods for recalibration is through supervised means, and unsupervised means. Supervised recalibration requires the prosthesis user to manually decide if the prosthesis is not performing optimally, and to collect a new training dataset for the device. The new set of training data will either be appended to the existing data or shall replace the previous training data, this retraining has often been done through screen guided training [16]. A prosthesis guided training method was proposed by Chicone et al [4], upon recalibration, the prosthesis would perform a series of gestures for the user to mimic. It was found that the prosthesis guided approach both provided better training samples and reduced the need for any additional devices to the prosthesis. Unsupervised methods, however, require no conscious input on the prosthesis users behalf. Adaptive algorithms are used to regularly update the existing training data based on different adaptive strategies. There has been successful research papers on improving classification accuracy with adaptive techniques $[13,14,20]$. An example would be Sensinger et al [15], noted that unsupervised methods that rely on high confidence of classification could provide implementable degrees of accuracy, yet could suffer over-training over long periods of time. It was further found that adaptive methods based on low confidence of classification could decrease over training but were not reliable when unsupervised.

Although Adaptive methods are very capable of improving the overall time between conscious calibration, and may subsequently provide higher degrees of long term accuracy, the data selection schemes are not fully dependable for clinical use. Two general problems must be addressed with selecting a training scheme. Firstly, in schemes that directly modify the training set for retraining, the overall size of the training dataset should be minimal as to avoid overtraining and to reduce the computation time during training. Secondly, that concept drift within EMG signals is not fully understand and therefore difficult to adequately model the change over time, or to capture a training data set that is representative of the range of gestures. Generally the selection of original data could be more important for initial use, largely due to the variable nature of EMG signals throughout a day.

Electromyography (EMG) is the method of analysing the electrical impulses from various muscles within the human body. Through pattern recognition, a machine may be trained to both understand and predict motions from monitored muscles. Although, in a laboratory setting, results are promising, clinical success of pattern recognition based prosthesis is still lacking. In academia, there exists increasingly promising methods for recognition of motion intent, with accuracy rates commonly reaching above $90 \%$. However, a large quantity of academic research focuses on training with offline datasets, whilst online classification results are not as satisfactory. There are several potential causes for decreased accuracy during online classification such as: skin-electrode impedance [17], electrode displacement and shift [19], and fatigue [12].

In this study, an investigation into methods of data selection to improve the long term inter-day stability of a trained dataset shall be conducted. The following 
sections shall cover data collection, data selection, and processing, before a discussion on the findings of this research, ending in a conclusion and suggestions towards future work.

\section{Methodology}

\subsection{Experimental setup}

Data collection device To collect EMG data, a 16 channel device was used with 12 bits ADC resolution and $1 \mathrm{Khz}$ sampling frequency. The range for collected EMG data was $10 \mathrm{~Hz}$ to $500 \mathrm{~Hz}$ through a band pass filter in the hardware, $50 \mathrm{~Hz}$ powerline noise was removed with a notch filter in the hardware and a comb filter in the software. The EMG data was transferred from the device via USB, and processed on a Windows based PC. More details may be found in the previous works $[8,7]$.

Device Wearing The devices structure is that of a sleeve with 18 embedded electrodes, with two bias electrodes. The exact configuration of electrodes within the sleeve are arranged as to improve the signal repeatability [5]. A second sleeve was worn over the electrode sleeve to ensure a tight fit on all electrodes. The sleeve was worn on the subjects dominant arm, or preferred arm in cases of Cross-dominance. To ensure each day saw the same fitting, a marker was used to denote the location of both bias electrodes on the subjects arm, in-line with the top and bottom of the sleeve, as shown in Fig.1a. The degree of shift experienced on the sleeve was updated by new markings before each instance of data collection. The benefit of the dual sleeve arrangement in this device is that it maintains both pressure and contact upon a larger section of the arm. Through the increased surface contact, the device is subsequently less likely to shift through and dramatic degree during wearing. During the course of this study it was found that once the sleeve had begun to imprint upon the skin, that any shift would gradually re-align with the original rotation on the arm. The flexible design of the elasticated fabric further meant that relative positions of the electrodes to the arm are very stable.

Issues such as sweating may be a factor in warm climates, or if the wearer is performing much physical activity, however, neither of these issues were encountered during the data collection. In a situation where the device was exposed to cold climates the wearer would allow the device to return to the ambient room temperature before data collection. The potential reasoning for this is that colder climates lead to a drier skin surface and subsequently high skin-electrode impedance.

Data Collection Data collection began at 10:00 for each day and ended at 18:00, a 30 minute window was granted between data collection providing a total of 16 sessions per day. the 30 minute window is designed to keep constant records of EMG data without placing unnatural fatigue on the subject. EMG 
capture was performed by the subject sitting in front of a computer monitor, with elbows in a fixed position. Thirteen separate hand gestures, as shown in Fig.1b, were shown to the subject with 5 seconds to transfer between gestures and 5 seconds of holding a gesture. The series of gestures were presented to the subject in a pseudo-random order, as to prevent the subject learning the order of gestures and transferring to a new gesture too early. The transition period both allows the participant to move between gestures and to have a steady gesture held for the next recording period.

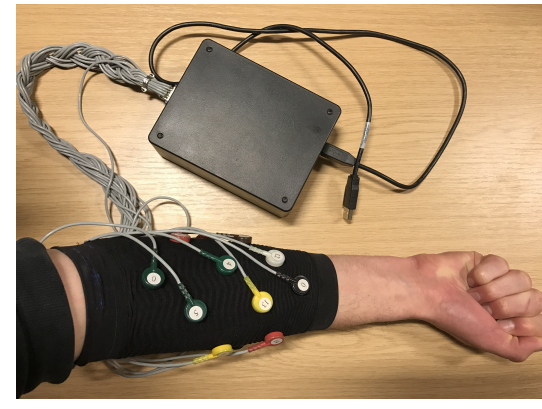

(a) Elonxi device

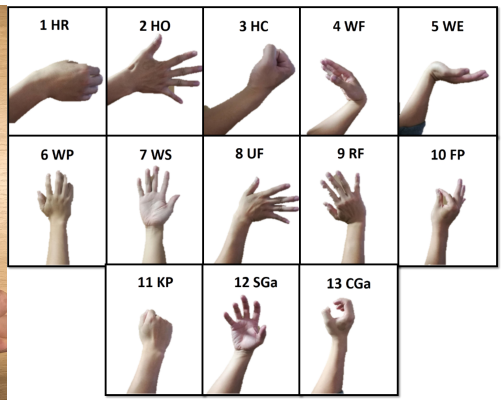

(b) Training gestures

Fig. 1. Elonxi device and training gestures

Data processing and classification Signal collection was performed through custom software, and processed in Matlab. As stated in the data collection section, the custom software would strip any remaining powerline noise and output what would hopefully be the EMG signal with minimal interference. Once the raw signal had been stripped of any interference and prepared for processing, the outputted EMG signal data was stripped of the transient signal between gestures. This is to say, the 5 second window of transition between each gesture was removed as to ensure each set of data is fully representative of representative class.

The feature extraction was performed in a $250 \mathrm{~ms}$ window, with a $50 \mathrm{~ms}$ sliding window, the window size was chosen as it . the chosen features for this study were a combination of Auto-Regressive (AR) to the fourth order and root Mean Square (RMS), as the combination of these features had previously been shown to be both strong features and robust for long term classification [3, 9]. For classification, Linear Discriminant Analysis (LDA) was used with a bayes-optimal classifier. Although Support Vector Machines (SVM) and Multi-Layer Perceptrons (MLP) are good algorithms for classification, LDA has previously been shown to be more robust during long term classification, although at a cost of native classification accuracy whilst MLP and SVM decayed rapidly [11]. 


\subsection{Experiments}

Data selection To compare the relative performance of each method of selecting data, several data selection strategies were evaluated using a single day as training data and alternative days as testing days, these strategies were firstly applied without any testing day data and secondly with one of the first recorded sessions from the testing day. To compare the efficiency of the selection strategies, two control tests shall be used. It was found by Jain et al [10] that a single trained classifier can degrade to $78.4 \pm 2.33 \%$, therefore the first control case shall be the performance a classifier trained from one of the first 3 sessions of the testing day. The second control case shall be a classifier trained with one of the first sessions of a prior day, to monitor the decay of using minimal prior data.

The selection rules used are as follows:

1. The first session of a prior day

2. The first 2 sessions of a prior day

3. The first 3 sessions of a prior day

4. The first 4 sessions of a prior day

5. All 16 sessions of a prior day

6. 3 sessions, randomly selected from 3 points of a prior day

7. 4 sessions, randomly selected from 4 space points of a prior day

8. A single session of a prior day

The first 3 selection cases are to observe the impact of adding sequential sessions into the training dataset. The second and third case also provide a direct comparison of sequential data selection, as opposed to representative data selection. The usage of an entire day (16 sessions) is to observe the effect of using a larger dataset to represent an entire day of EMG signals.

The final non control cases are both within the proposed method of representative data selection. In this method, a given day is separated into descriptive periods such as: morning, middle day, and evening. The intention of this method is to provide a good representation of the shifts in the EMG signal during any given day whilst using a minimal quantity of recording points in aforementioned day. An example of selection of random data points for training data is demonstrated in Fig.2.

Data Analysis As mentioned in the Data selection segment, several strategies of data selection are to be tested. As this study focuses on the general performance of the data selection strategies, the temporal change in accuracy along consecutive days has not been recorded. Instead, each day in the dataset is considered as a unique training day against all other days which shall be considered as testing days, without considering for their appearance in sequence. Each training day will therefore be tested against every other day using the different selection strategies, each test shall begin with the first session of the testing day and then continue until the final session, recording the accuracy of each internal session. This process shall be repeated for every other day until 


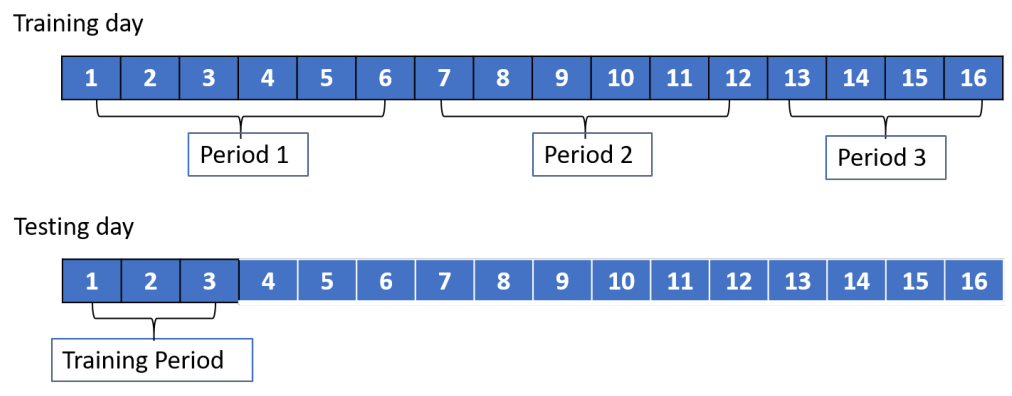

Fig. 2. Proposed Selection Rule using 3 prior day sessions and one testing day session

the accuracy of every training method on every possible training day has been collected. The final resulting interday accuracy shall then be calculated as the outputted mean when computing firstly the mean of each testing days internal performance, and then acquiring the mean of each testing days final performance. Ideally the output of acquiring the overall mean performance will grant insight into the generalisation of these methods.

\section{Results}

Fig.3 shows the averaged interday performance of the four data selection methods, all are shown with and without a testing day session whereas Table.1 shows the complete list of accuracy and the standard deviation of each method. As demonstrated by other authors [18], the interday accuracy of a single training set is very poor at $61.9 \%$, although this level of accuracy is very similar to that of the intraday accuracy, as an early morning session alone is unable to fully represent transient changes within the EMG signal.

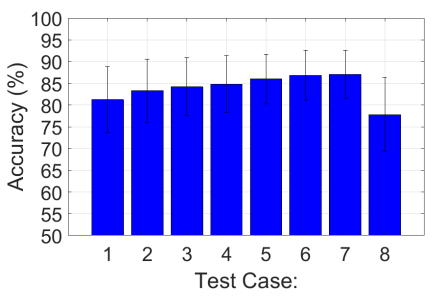

(a) With Testing Day Data

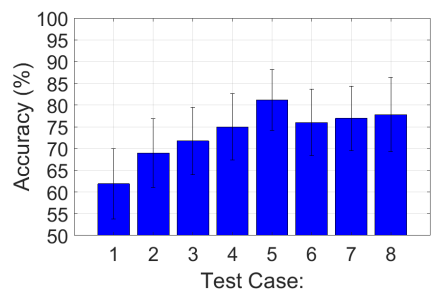

(b) Without Testing Day Data

Fig. 3. Results of all data selection rules listed. Case 1: First session only Case 2: First 2 sessions, Case 3: First 3 sessions Case 4: First 4 sessions Case 5: Entire Day (16 Sessions), Case 6: 3 Spread out sessions, Case 7: 4 Spread out sessions, Case 8: Single Session from testing day only 
Table 1. Results of using sessions from prior day and first session of testing day as training data against the entirety of the testing day

\begin{tabular}{|l|l|l|}
\hline Classification Results & Without Testing Day Data & With Testing Day Data \\
\hline Training Day Data: & Accuracy(\%) & Accuracy(\%): \\
\hline Testing Day data only & $77.8 \pm 8.6$ & $77.8 \pm 8.6$ \\
\hline Session 1 & $61.9 \pm 8.0$ & $81.2 \pm 7.6$ \\
\hline Session 1-2 & $68.3 \pm 7.9$ & $83.3 \pm 7.3$ \\
\hline Sessions 1-3 & $71.7 \pm 7.8$ & $84.2 \pm 6.7$ \\
\hline Sessions 1-4 & $75.0 \pm 7.8$ & $84.8 \pm 6.5$ \\
\hline Entire day of 16 sessions & $81.2 \pm 7.0$ & $86.0 \pm 5.7$ \\
\hline 3 Spaced Sessions & $76.0 \pm 7.6$ & $86.9 \pm 5.7$ \\
\hline 4 Spaced sessions & $76.9 \pm 7.4$ & $87.0 \pm 5.5$ \\
\hline
\end{tabular}

The other selection methods managed to achieve constantly acceptable accuracy rates of $85 \%+-2 \%$ accuracy on interday testing sets. Showing that a single days worth of data is capable of providing a good representation of a persons EMG signals.

The highest result was found with using the proposed methods of the selecting defining periods of each training day with a single early session of the testing day with an accuracy of $86.9 \% \pm 5.7 \% 87.0 \% \pm 5.5 \%$, for both the 3 and 4 sessions. When the training set has prior knowledge of the testing day, the result reduces to $76.0 \% \pm 7.6 \%$ and $76.9 \% \pm 7.4 \%$ which is very similar to using the first session alone of any given day (77.8\%). The results of the entire day could be considered to be the effect of gathering a large enough dataset to accurately represent the gradual change of the signal during an average day. Using 4 morning sessions managed achieve a reasonable amount of accuracy, although the deviation implies that it may not be fully acceptable for daily usage, the accuracy also saw a large decrease when not using a testing day dataset. Finally, using 3 spaced datasets and 1 testing day set as training data resulted in similar accuracy to the entire day training set.

As shown in the confusions matrix in Fig.4a, the proposed method achieved a high degree of accuracy on the majority of simple gestures, where the average inter-day accuracy was 90\%, which poses itself as a very strong method for reliable long term EMG prosthesis control. Generally, the majority of simple classes were easily classified. The classes which routinely scored most poorly were the two complex gestures numbered 12 and 13 in Fig.1b, where accuracy was rarely above $70 \%$ in any test case.

\section{Discussion}

In this study, the effect of different training dataset selection strategies was investigated. The results demonstrated a stronger classifier, for inter-day EMG gesture recognition, can be built by using data that is representative of the EMG signal changes within a day. 


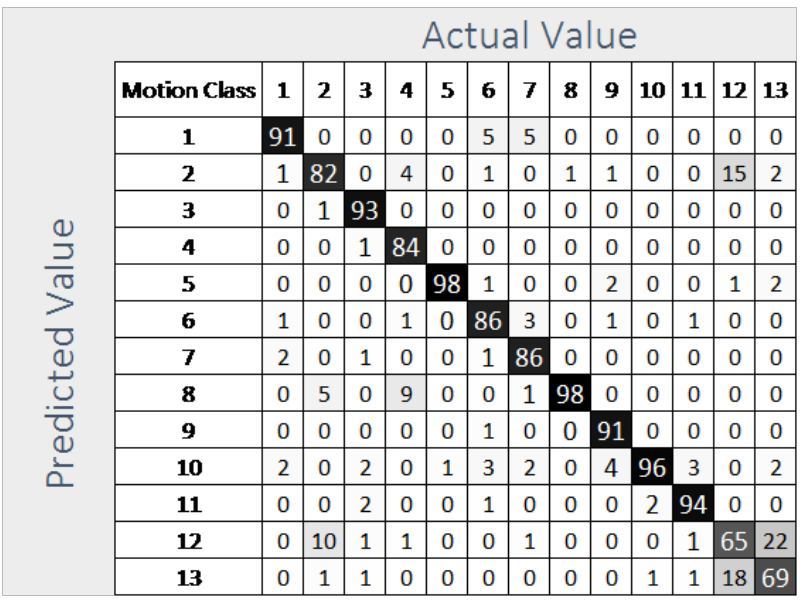

(a) Confusion Matrix for Proposed Method of 3 Spaced Data Points

\begin{tabular}{|c|c|c|c|c|c|c|c|c|c|c|c|c|c|c|}
\hline & \multicolumn{14}{|c|}{ Actual Value } \\
\hline \multirow{14}{*}{ 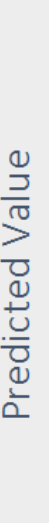 } & Motion Class & 1 & 2 & 3 & 4 & 5 & 6 & 7 & 8 & 9 & 10 & 11 & 12 & 13 \\
\hline & 1 & 93 & 0 & 0 & 0 & 0 & 6 & 5 & 0 & 0 & 0 & 0 & 0 & 0 \\
\hline & 2 & 1 & 82 & 0 & 5 & 0 & 1 & 0 & 1 & 1 & 0 & 0 & 16 & 1 \\
\hline & 3 & 0 & 1 & 86 & 0 & 0 & 0 & 0 & 0 & 0 & 0 & 0 & 0 & 0 \\
\hline & 4 & 0 & 0 & 1 & 79 & 0 & 1 & 0 & 0 & 0 & 0 & 0 & 0 & 0 \\
\hline & 5 & 0 & 0 & 0 & 0 & 95 & 1 & 0 & 0 & 4 & 0 & 0 & 0 & 2 \\
\hline & 6 & 1 & 0 & 0 & 2 & 0 & 85 & 2 & 0 & 1 & 0 & 1 & 0 & 0 \\
\hline & 7 & 1 & 0 & 0 & 0 & 0 & 2 & 88 & 0 & 0 & 0 & 0 & 0 & 0 \\
\hline & 8 & 0 & 2 & 0 & 11 & 0 & 0 & 1 & 97 & 0 & 0 & 0 & 0 & 0 \\
\hline & 9 & 0 & 0 & 0 & 0 & 1 & 1 & 0 & 0 & 88 & 1 & 0 & 0 & 0 \\
\hline & 10 & 1 & 1 & 2 & 0 & 3 & 3 & 2 & 0 & 6 & 96 & 6 & 0 & 1 \\
\hline & 11 & 0 & 0 & 4 & 0 & 0 & 1 & 0 & 0 & 0 & 1 & 90 & 0 & 0 \\
\hline & 12 & 0 & 12 & 6 & 1 & 0 & 0 & 1 & 0 & 0 & 0 & 1 & 60 & 17 \\
\hline & 13 & 0 & 2 & 0 & 0 & 0 & 0 & 0 & 0 & 0 & 1 & 1 & 23 & 76 \\
\hline
\end{tabular}

(b) Confusion Matrix for Entire Day of Data

\begin{tabular}{|c|c|c|c|c|c|c|c|c|c|c|c|c|c|c|}
\hline & \multicolumn{14}{|c|}{ Actual Value } \\
\hline \multirow{14}{*}{ 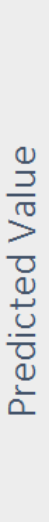 } & Motion Class & 1 & 2 & $\mathbf{3}$ & 4 & 5 & 6 & 7 & 8 & 9 & 10 & 11 & 12 & 13 \\
\hline & 1 & 71 & 0 & 0 & 0 & 0 & 3 & 5 & 0 & 0 & 0 & 0 & 0 & 0 \\
\hline & 2 & 2 & 63 & 0 & 6 & 0 & 4 & 1 & 2 & 1 & 0 & 0 & 5 & 1 \\
\hline & 3 & 0 & 1 & 83 & 1 & 0 & 0 & 0 & 0 & 0 & 0 & 2 & 0 & 0 \\
\hline & 4 & 0 & 0 & 2 & 76 & 0 & 1 & 0 & 0 & 0 & 0 & 0 & 0 & 0 \\
\hline & 5 & 0 & 0 & 0 & 1 & 98 & 1 & 0 & 0 & 11 & 4 & 1 & 1 & 16 \\
\hline & 6 & 2 & 0 & 0 & 1 & 0 & 83 & 4 & 0 & 2 & 0 & 1 & 0 & 0 \\
\hline & 7 & 6 & 0 & 0 & 0 & 0 & 0 & 75 & 0 & 0 & 0 & 0 & 0 & 0 \\
\hline & 8 & 0 & 13 & 0 & 10 & 0 & 0 & 2 & 97 & 0 & 2 & 5 & 2 & 5 \\
\hline & 9 & 0 & 0 & 0 & 0 & 0 & 1 & 1 & 0 & 76 & 0 & 1 & 0 & 0 \\
\hline & 10 & 17 & 0 & 3 & 0 & 1 & 6 & 11 & 0 & 9 & 91 & 5 & 0 & 4 \\
\hline & 11 & 0 & 0 & 1 & 0 & 0 & 0 & 0 & 0 & 0 & 1 & 80 & 0 & 0 \\
\hline & 12 & 0 & 22 & 2 & 4 & 0 & 0 & 0 & 1 & 1 & 0 & 3 & 73 & 27 \\
\hline & 13 & 0 & 2 & 8 & 0 & 1 & 0 & 1 & 0 & 0 & 1 & 1 & 18 & 44 \\
\hline
\end{tabular}

(c) Confusion Matrix for Single Testing Day Session

Fig. 4. Confusion Matrices of selected Approaches with testing day data 
The single sessions accuracy and reduction in accuracy when excluding a testing day dataset demonstrates the previous conclusion by Amsuss et al [2] that classification accuracy decreases as a function of time. The accuracy of using 4 morning sessions is directly inline with those displayed by Kaufmann [11] when using LDA, where the error rate they had found was $21.27 \%$, whilst this study found an error rate of $25 \% \pm 7.8 \%$ when using their first 5 sessions. Unfortunately it was Kaufmann had not noted which periods of each day saw data collection, only that 5 datasets represented an entire day of data, whereas this study focused on the impact of when the data is collected within a day. However, when given any set of 4 consecutive hours provided similar performance on inter-day trials, suggesting that any 4 consecutive sessions will provide a seemingly reliable description of the EMG signal. It can further be agreed that using larger quantities of data, such as an entire day of data, would provide higher classification accuracy, however, the increase in accuracy is not statistically significant enough to justify the increase in computation and burden on the user.

Although the proposed method, with no prior knowledge of the testing day, achieves a similar degree of accuracy to training the algorithm in the morning of the testing day, the removal of the requirement to train the device immediately and achieve a reasonable degree of accuracy is very promising. Through the addition of the testing day data, the proposed method provided a very good inter-day accuracy without any modification to the training dataset. The extent of the inter-day accuracy is one where it could be expected to achieve high accuracy on future datasets, regardless of time difference between the original training day and present day.

As shown in the confusion matrices in Fig.4, the simple gestures routinely were easy to classify from each test case, or at least represented the final accuracy best. The two complex gestures routinely scored very low, often being misclassified as one another. Due to this drop in accuracy being so localized, it could be suggested that using this method without those two classes could be more beneficial for the prosthesis user. The researcher is, however, of the opinion that it is important to promote a prosthesis control scheme which is both natural and intuitive for the amputee. Regarding the actual outputted results, differing methods would either provide higher accuracy on one complex gesture at the cost of reduced accuracy on the other gesture. Although the performance of the proposed algorithm for complex gestures is still not fully adequate for control, it displays a promising removal of the bias for either gesture. The usage of improved feedback methods during data collection may assist a prosthesis user in providing more and clear separable data for the complex gestures [6].

It should be noted that although the performance of this method is good for the majority of presented data, it has not been tested against fatigued data or data where the presence of shift exists. Subsequently, the robustness of this method has not yet been shown to be resilient to transient changes other than those which naturally throughout a day or across a period of time. 


\section{Conclusion}

In this paper, the relationship between the periods of a day and the EMG signal was investigated. From the results of this preliminary study, there lies two main conclusions. Firstly, that selection of a data that can represent the change in the EMG signal throughout a standard day is capable of achieving an acceptable degree of accuracy in the long term. Further to this point, it can be assumed that such data must be fully representative of the day itself, at the risk of losing stability.

Secondly, the selection strategies in this paper suggest that usage of an entire days data provides a statistically insignificant increase in classification accuracy when compared to more simplistic and computationally efficient methods.

The intent of this research was to investigate the changes in the EMG signal during a day, its impact on classification accuracy over a series of days, and to

find a method that was suited to promoting a robust training set for inter-day classification throughout each day.

It is suggested that future directions to expand upon this research is to look at ways of optimising or otherwise processing the original training dataset to better describe the shift in the EMG signal, either through promoting expansion or promoting clustering. Further cases of study may also investigate the relative change in performance with days that experience high degrees of fatigue or other transient changes during a single day, where external changes may impact the quality of the EMG signal.

\section{References}

1. Amsss, S., Paredes, L.P., Rudigkeit, N., Graimann, B., Herrmann, M.J., Farina, D.: Long term stability of surface emg pattern classification for prosthetic control. In: 2013 35th Annual International Conference of the IEEE Engineering in Medicine and Biology Society (EMBC). pp. 3622-3625 (July 2013)

2. Amsuss, S., Paredes, L.P., Rudigkeit, N., Graimann, B., Herrmann, M.J., Farina, D., Subjects, A.: Long Term Stability of Surface EMG Pattern Classification for Prosthetic Control * pp. 3622-3625 (2013)

3. Chen, X., Zhang, D., Zhu, X.: Application of a self-enhancing classification method to electromyography pattern recognition for multifunctional prosthesis control. Journal of neuroengineering and rehabilitation pp. 1-13 (2013)

4. Chicoine, C.L., Simon, A.M., Hargrove, L.J.: Prosthesis-guided training of pattern recognition-controlled myoelectric prosthesis. In: 2012 Annual International Conference of the IEEE Engineering in Medicine and Biology Society. pp. 1876-1879 (Aug 2012)

5. Fang, Y., Liu, H.: Robust semg electrodes configuration for pattern recognition based prosthesis control. In: 2014 IEEE International Conference on Systems, Man, and Cybernetics (SMC). pp. 2210-2215 (Oct 2014)

6. Fang, Y., Zhou, D., Li, K., Liu, H.: Interface prostheses with classifier-feedback based user training. IEEE Transactions on Biomedical Engineering PP(99), 1-1 (2017) 
7. Fang, Y., Liu, H., Li, G., Zhu, X.: A multichannel surface emg system for hand motion recognition. International Journal of Humanoid Robotics 12(02), 1550011 (2015), http://www.worldscientific.com/doi/abs/10.1142/S0219843615500115

8. Fang, Y., Zhu, X., Liu, H.: Development of a surface emg acquisition system with novel electrodes configuration and signal representation. In: International Conference on Intelligent Robotics and Applications. pp. 405-414. Springer (2013)

9. Huang, Y., Englehart, K.B., Hudgins, B., Chan, A.D.C.: A Gaussian mixture model based classification scheme for myoelectric control of powered upper limb prostheses. IEEE Transactions on Biomedical Engineering 52(11), 1801-1811 (2005)

10. Jain, S., Singhal, G., Smith, R.J., Kaliki, R., Thakor, N.: Improving long term myoelectric decoding, using an adaptive classifier with label correction. In: Proceedings of the IEEE RAS and EMBS International Conference on Biomedical Robotics and Biomechatronics. pp. 532-537 (2012)

11. Kaufmann, P., Englehart, K., Platzner, M.: Fluctuating EMG Signals : Investigating Long-term Effects of Pattern Matching Algorithms pp. 6357-6360 (2010)

12. Lalitharatne, T.D., Hayashi, Y., Teramoto, K., Kiguchi, K.: Compensation of the effects of muscle fatigue on emg-based control using fuzzy rules based scheme. In: 2013 35th Annual International Conference of the IEEE Engineering in Medicine and Biology Society (EMBC). pp. 6949-6952 (July 2013)

13. Orabona, F., Castellini, C., Caputo, B., Fiorilla, A.E., Sandini, G.: Model adaptation with least-squares svm for adaptive hand prosthetics. Idiap-RR Idiap-RR-052009, Idiap (3 2009), accepted in ICRA09

14. Pilarski, P.M., Dawson, M.R., Degris, T., Carey, J.P., Chan, K.M., Hebert, J.S., Sutton, R.S.: Adaptive artificial limbs: a real-time approach to prediction and anticipation. IEEE Robotics Automation Magazine 20(1), 53-64 (March 2013)

15. Sensinger, J.W., Lock, B.A., Kuiken, T.A.: Adaptive pattern recognition of myoelectric signals: Exploration of conceptual framework and practical algorithms. IEEE Transactions on Neural Systems and Rehabilitation Engineering 17(3), 270278 (June 2009)

16. Simon, A.M., Hargrove, L.J., Lock, B.A., Kuiken, T.A.: A decision-based velocity ramp for minimizing the effect of misclassifications during real-time pattern recognition control. IEEE Transactions on Biomedical Engineering 58(8), 2360-2368 (Aug 2011)

17. Vidovic, M.M.C., Hwang, H.J., Amsss, S., Hahne, J.M., Farina, D., Mller, K.R.: Improving the robustness of myoelectric pattern recognition for upper limb prostheses by covariate shift adaptation. IEEE Transactions on Neural Systems and Rehabilitation Engineering 24(9), 961-970 (Sept 2016)

18. Vidovic, M.M., Hwang, H.j., Amsüss, S., Hahne, J.M., Farina, D., Müller, K.r.: Improving the Robustness of Myoelectric Pattern Recognition for Upper Limb Prostheses by Covariate Shift Adaptation 24(9), 961-970 (2016)

19. Young, A.J., Hargrove, L.J., Kuiken, T.A.: The effects of electrode size and orientation on the sensitivity of myoelectric pattern recognition systems to electrode shift. IEEE Transactions on Biomedical Engineering 58(9), 2537-2544 (Sept 2011)

20. Zhang, Y., Wang, Z., Zhang, Z., Fang, Y., Liu, H.: Comparison of online adaptive learning algorithms for myoelectric hand control. In: 2016 9th International Conference on Human System Interactions (HSI). pp. 69-75 (July 2016) 